\title{
Fusion Sign of Tendon-to-Bone Healing on $X$-Ray after Rotator Cuff Repair
}

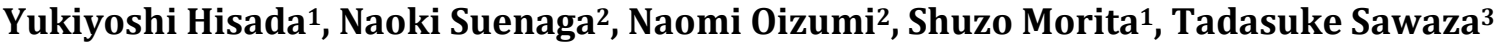 \\ ${ }^{1}$ Department of Orthopaedic Surgery, Kaisei Hospital, Obihiro, Hokkaido, Japan \\ ${ }^{2}$ Upper Extremity Center of Joint Replacement and Endoscopic Surgery, Hokushin Orthopaedic Hospital, Sapporo, Hokkaido, Japan \\ ${ }^{3}$ Department of Radiology, Kaisei hospital, Obihiro, Hokkaido, Japan \\ Email: hygti1967jp-fs@yahoo.co.jp
}

How to cite this paper: Hisada, Y., Suenaga, N., Oizumi, N., Morita, S. and Sawaza, T. (2021) Fusion Sign of Tendon-to-Bone Healing on X-Ray after Rotator Cuff Repair. Open Journal of Orthopedics, 11, 22-31. https://doi.org/10.4236/ojo.2021.111003

Received: December 13, 2020

Accepted: January 23, 2021

Published: January 26, 2021

Copyright (c) 2021 by author(s) and Scientific Research Publishing Inc. This work is licensed under the Creative Commons Attribution International License (CC BY 4.0).

http://creativecommons.org/licenses/by/4.0/

\section{(c) (i) Open Access}

\begin{abstract}
Purpose: In clinical practice, increased radiolucency at the insertion site of a repaired tendon on the humerus on postoperative radiographs of patients following rotator cuff repair is often observed. Separately, magnetic resonance imaging (MRI) revealed tendon-to-bone healing in conjunction with this finding. Thus, we suspected that such radiographic changes are associated with tendon-to-bone healing, a phenomenon we labeled as the "fusion sign." This study sought to investigate the diagnosis rate of the fusion sign in relation to tendon-to-bone healing after rotator cuff repair. Methods: Patients who underwent open rotator cuff repair (ORCR) or arthroscopic rotator cuff repair (ARCR) at two centers from 2010 to 2018 and who underwent MRI more than 6 months postoperatively were included in this study. The presence of radiolucency of the humeral footprint on a radiograph (the fusion sign) was investigated and checked for the concurrent presence of tendon-to-bone healing on MRI. Results. In total, 187 shoulders after ARCR and 55 shoulders after ORCR were included in this study. Among these, SH repair was performed in 202 shoulders and suture-bridging repair was performed in 40 shoulders. The fusion sign was positive in $67.8 \%$ of cases and negative in $32.3 \%$. The positive predictive value (PPV) of the total population was 0.963 and did not differ according to the suture method used, reported as 0.964 in ARCR, 0.962 in ORCR, 0.966 in the surface-holding technique, and 0.938 in the suture-bridging technique. The intraobserver reliability was $\kappa=0.4478(p<0.001)$. The interobserver reliability for all observers was $\kappa=0.408(p<0.001)$. Conclusion: Postoperatively, the presence of the fusion sign at the footprint of the humerus strongly suggests that tendon-to-bone healing has occurred.
\end{abstract}

\section{Keywords}

Rotator Cuff, Orthopedic Procedures, X-Ray, Magnetic Resonance Imaging 


\section{Introduction}

Rotator cuff tendon tears are very common, with incidence rates of $15 \%-20 \%$ among individuals aged 60 years, $26 \%-30 \%$ among individuals aged 70 years, and 36\% - 50\% among individuals aged 80 years [1] [2]. Tendon tears may cause significant pain and reduce functional performance. Open or arthroscopic repairs of full-thickness rotator cuff tears have included an increasing number of orthopedic procedures in recent years.

Magnetic resonance imaging (MRI) and ultrasonography (US) are the most commonly used imaging methods to confirm tendon-to-bone healing postoperatively. However, MRI and US cannot be deployed in all facilities, have certain cost and time requirements, and cannot be performed often. Conversely, plain $\mathrm{X}$-rays are easier to apply as a routine examination in any medical institution. The ability to accurately predict tendon-to-bone healing using X-rays would be beneficial for patients. However, to our knowledge, there has been no previous study that proved an association between tendon-to-bone healing and X-ray findings.

In clinical practice, increased radiolucency at the insertion site of the repaired tendon on the humerus in postoperative radiographs was often found (Figure 1(A), Figure 1(B), Figure 2(A) and Figure 2(B)). When MRI is performed in these cases, tendon-to-bone healing is often revealed (Figure 1(C) and Figure 2(C)); thus, we came to suspect that those radiographic changes are associated with tendon-to-bone healing. We ultimately named this kind of radiographic change as a "fusion sign."

Subsequently, in the present study, we hypothesized that the presence of radiolucency at the humeral footprint in an X-ray is a sign of tendon-to-bone healing after rotator cuff repair. This study aimed to investigate the diagnostic rate and interobserver and intraobserver reliabilities of the fusion sign in correlation with tendon-to-bone healing after rotator cuff repair.

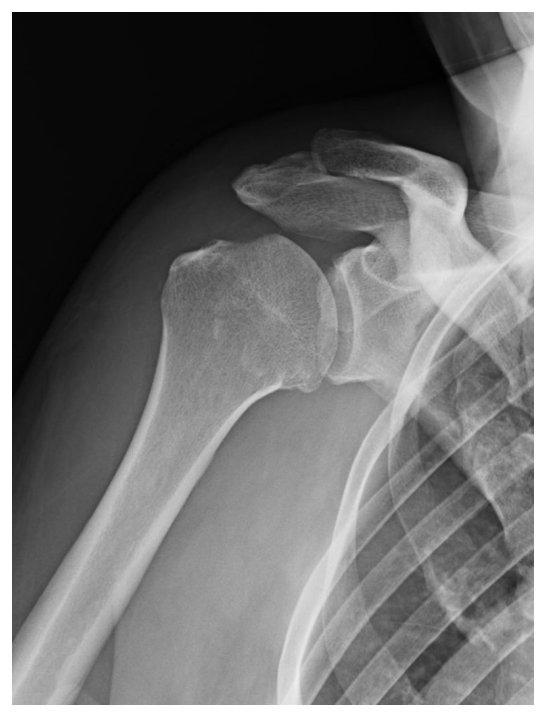

(A) 


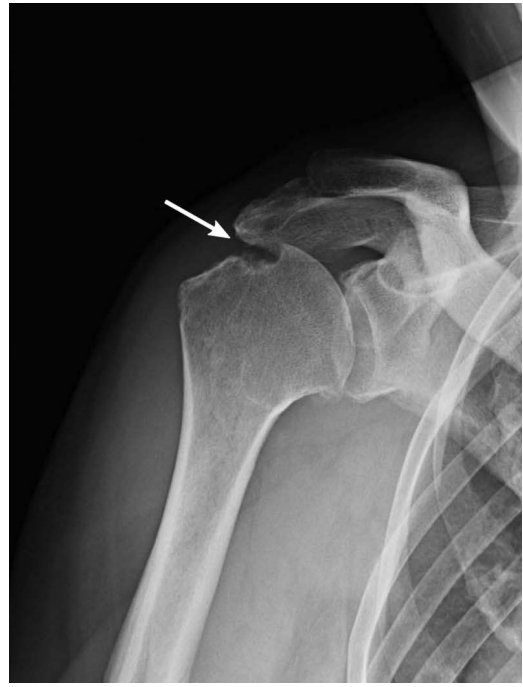

(B)

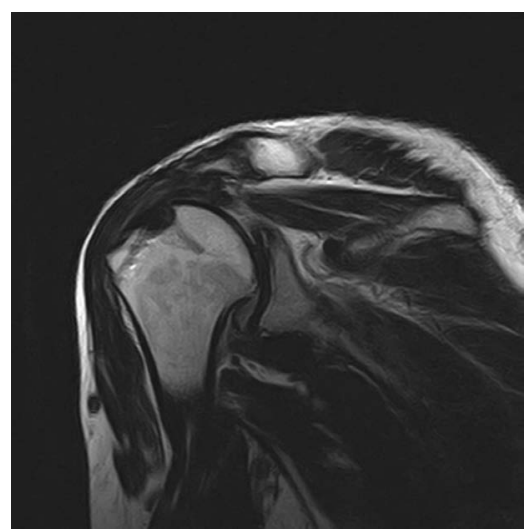

(C)

Figure 1. (A) Anteroposterior (AP) radiograph of a right shoulder 1 week after rotator cuff repair (ORCR). (B) The fusion sign (arrow). The AP radiograph demonstrates radiolucency at the insertion site of repaired tendon on the humerus at 9 months postoperatively. (C) Tendon-to-bone healing is visible on magnetic resonance imaging at 9 months postoperatively.

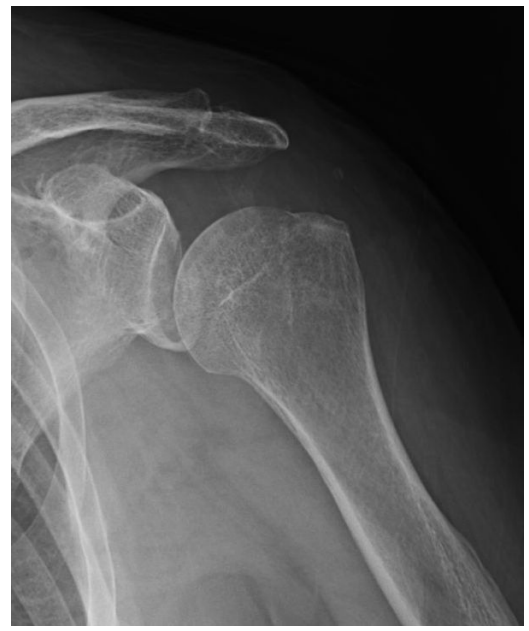

(A) 


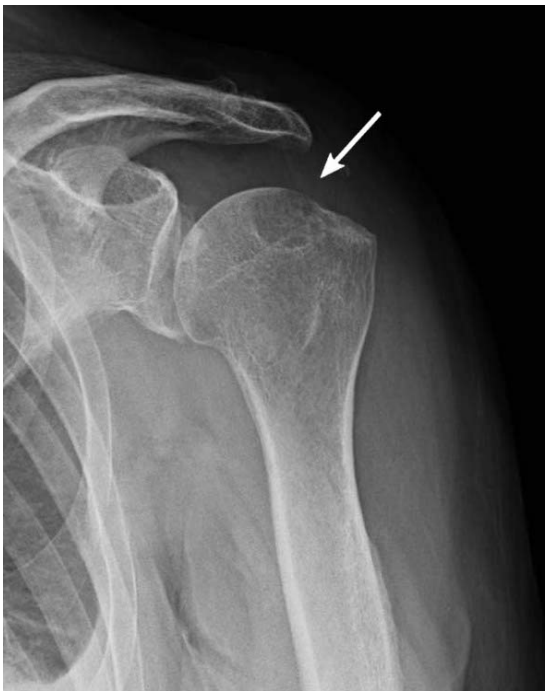

(B)

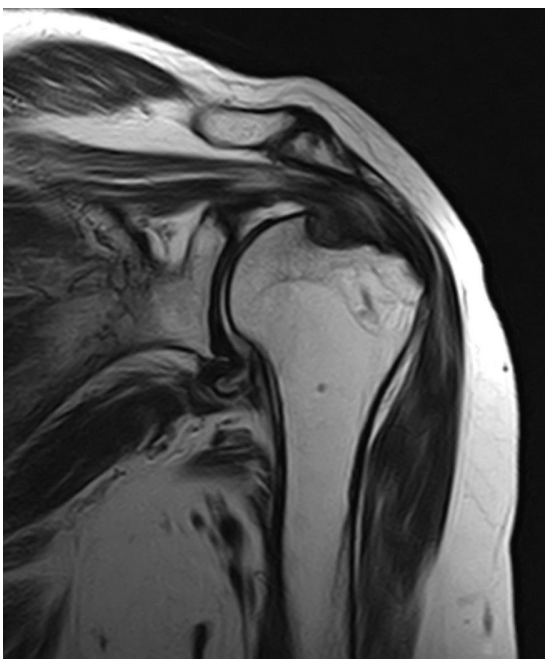

(C)

Figure 2. (A) Anteroposterior (AP) radiograph of a left shoulder 1 week after rotator cuff repair (ARCR). (B) The fusion sign (arrow). The AP radiograph demonstrates radiolucency at the insertion site of repaired tendon on the humerus at 10 months postoperatively. (C) Tendon-to-bone healing is visible on magnetic resonance imaging at 10 months postoperatively.

\section{Materials and Methods}

This retrospective interventional study included patients who underwent open rotator cuff repair (ORCR) or arthroscopic rotator cuff repair (ARCR) at Kaisei Hospital and Kushiro Sanjikai Hospital from 2010 to 2018 and who underwent MRI $>6$ months postoperatively. The present study was approved by the institutional review board at Hokushin Orthopaedic Hospital (no. 1905). All surgeries were performed by one surgeon (co-author, N. S.) at both centers. Tears were classified intraoperatively using the system described by DeOrio and Cofield [3] as follows: small, $<1 \mathrm{~cm}$; medium, $1-3 \mathrm{~cm}$; large, $3-5 \mathrm{~cm}$; and massive, $>5$ $\mathrm{cm}$ or involving two tendons. Patients with small to medium tears underwent 
arthroscopic suture bridge (SB) repair [4] or surface-holding (SH) repair [5] [6], whereas those with large to massive tears underwent repair using the arthroscopic or open $\mathrm{SH}$ technique. The $\mathrm{SH}$ technique is a modified transosseous-equivalent procedure using medial anchors and lateral transosseous sutures, as we reported previously [5] [6]. In total, 187 shoulders after ARCR and 55 shoulders after ORCR were included in this study. Among these, SH repair was performed in 202 shoulders and suture-bridging repair was performed in 40 shoulders.

Surgical procedure

All patients underwent preoperative interscalene block under general anesthesia. All procedures were performed with the patients lying in the beach chair position. During the surgical procedure, acromioplasty was performed with the resection of the coracoacromial ligament as described by Ellman [7]. In both surgical methods [5] [6], the footprint was created by removing cortical and subchondral bone until the cancellous bone was completely exposed. Bone marrow stimulation was also performed at the footprint [8] [9] [10]. Following adequate mobilization of the torn tendon, the tendon was repaired at the footprint under no excessive tension with the arm positioned at the side.

Postoperatively, an abduction brace was used for 6 weeks for small to large tears or 8 weeks in some cases presenting massive tears. Further, a systematic postoperative rehabilitation program was conducted with self-assisted range-of-motion exercises at 2 weeks postoperatively. Active elevation in the sitting position from the adducted shoulder position was permitted after 8 - 10 weeks, while isometric cuff exercises were initiated at $10-12$ weeks for those patients with medium or large massive tears, respectively. Patients were allowed to return to heavy work or sports after passing an assessment of sufficient muscle strength and range-of-motion recovery at 6 months postoperatively.

For each patient, we investigated the presence of radiolucency of the footprint on an X-ray (the "fusion sign") and checked for the presence of tendon-to-bone healing using MRI. Plain X-rays (anteroposterior view) in neutral rotation were taken at 1 week postoperatively and the same day that MRI was performed $>6$ months later. The immediately postoperative X-ray was compared with the $\mathrm{X}$-ray taken later in concurrence with the MRI investigation; when radiolucency on the footprint of the tendon was observed, it was defined as a positive fusion sign, while, when there was no radiolucency, such was defined as a negative fusion sign. MRI (T2-weighted; coronal, axial, and sagittal views) was taken at $>6$ months postoperatively. According to Sugaya's classification [11], types 1, 2, and 3 were defined as tendon-to-bone healing.

For interobserver and intraobserver reliability, examiner A (Y. H., a shoulder surgeon) examined the $\mathrm{X}$-rays to determine the presence or absence of a fusion sign. Three months later, examiner A observed the same radiographs and reconfirmed the fusion sign where appropriate. In addition, a radiologist (examiner $\mathrm{B}$ ) and a general orthopedic surgeon (examiner $\mathrm{C}$ ) looked at the same X-rays to determine the presence or absence of the fusion sign. Intraobserver and interob- 
server reliabilities were then calculated.

\section{Statistical analysis}

To assess diagnostic accuracy, the sensitivity (Se), specificity (Sp), positive predictive value (PPV), negative predictive value (NPV), false-positive rate (FPR), and false-negative rate (FNR) were calculated. Interobserver and intraobserver reliabilities were reported using Cohen's kappa coefficients and Fleiss's kappa coefficients. All tests were two-tailed, and statistical significance was defined as when $p<0.05$. All statistical analyses were performed using $\mathrm{R}$ version 3.2.4 (R Foundation for Statistical Computing, Vienna, Austria).

\section{Results}

Between January 2010 and December 2018, 242 shoulders underwent rotator cuff repair at two centers and underwent MRI $>6$ months postoperatively. The characteristics of the patients were shown in Table 1.

The fusion sign was positive in $67.8 \%$ of cases and negative in $32.3 \%$. On MRI, $93.8 \%$ of patients were diagnosed as presenting a healed tendon, while $6.2 \%$ showed retears.

The diagnostic accuracy results as compared with MRI for observer A were 69.6\% Se, 60.0\% Sp, 96.3\% PPV, 11.5\% NPV, 40.0\% FPR, and 30.4\% FNR for the first time (A1) and 70.5\% Se, 26.7\% Sp, 93.6\% PPV, 5.6\% NPV, 73.3\% FPR, and 29.5\% FNR for the second time (A2) 3 months later (Table 2). For observer B, the results of 59.5\% Se, 26.7\% Sp, 92.5\% PPV, 4.2\% NPV, 73.3\% FPR, and 40.5\% FNR were obtained. Finally, for observer C, the results of $63.9 \% \mathrm{Se}, 33.3 \% \mathrm{Sp}$, 93.5\% PPV, 5.7\% NPV, 66.7\% FPR, and 36.1\% FNR were obtained (Table 2). The results for each surgical procedure and suture method are shown in Table 2 and Table 3. There was no significant difference between ARCR and ORCR (Table 3) and between SH repair and SB repair (Table 4).

The intraobserver reliability for the two passes made by observer $\mathrm{A}$ was $\kappa=$ $0.4478(p<0.001)$ (Table 5$)$, whereas the interobserver reliability for all observers

Table 1. Patient characteristics.

\begin{tabular}{cc}
\hline $\mathrm{n}$ & 242 \\
\hline Mean age (yrs: range) & $65.1(36-86)$ \\
Gender & \\
male/female & $141 / 101$ \\
Operated side & $151 / 91$ \\
right/left & \\
Tear size & 35 \\
small & 94 \\
medium & 37 \\
large & 76 \\
massive &
\end{tabular}


Table 2. Diagnostic accuracy of all observers.

\begin{tabular}{ccccccc}
\hline & Se & Sp & PPV & NPV & FPR & FNR \\
\hline A1 & 69.6 & 60.0 & 96.3 & 11.5 & 40.0 & 30.4 \\
A2 & 70.5 & 26.7 & 93.6 & 5.6 & 73.3 & 29.5 \\
B & 59.5 & 26.7 & 92.5 & 4.2 & 73.3 & 40.5 \\
C & 63.9 & 33.3 & 93.5 & 5.7 & 66.7 & 36.1 \\
\hline
\end{tabular}

FNR, false negative rate; FPR, false positive rate; NPV, negative predictive value; PPV, positive predictive value; Se, sensitivity; Sp, specificity.

Table 3. Comparison of arthroscopic rotator cuff repair and open rotator cuff repair.

\begin{tabular}{ccc}
\hline & к-value & 95\% confidence interval \\
\hline Arthroscopic rotator cuff repair & 0.113 & -0.072 to 0.300 \\
Open rotator cuff repair & 0.033 & -0.364 to 0.431 \\
\hline
\end{tabular}

No significant difference was detected.

Table 4. Comparison of surface-holding repair and suture bridge repair.

\begin{tabular}{ccc}
\hline & к-value & 95\% confidence interval \\
\hline Surface-holding repair & 0.139 & -0.057 to 0.337 \\
Suture bridge repair & -0.013 & -0.298 to 0.272 \\
\hline
\end{tabular}

No significant difference was noted.

Table 5. Intraobserver and interobserver reliabilities.

\begin{tabular}{cccc}
\hline & א-value & $95 \%$ confidence interval & P value $^{*}$ \\
\hline Intraobserver reliability & 0448 & $0.326-0.569$ & $<0.001$ \\
Interobserver reliability & & & $<0.001$ \\
A/B & 0.447 & $0.331-0.563$ & $<0.001$ \\
A/C & 0.403 & $0.283-0.524$ & $<0.001$ \\
B/C & 0.377 & $0.258-0.497$ & \\
\hline
\end{tabular}

*P values were determined using Cohen's kappa.

was $\kappa=0.408(p<0.001)$ (Table 5). Meanwhile, the kappa value between the two observers was $\kappa=0.447(p<0.001)$ for observers A (first time) and $\mathrm{B}, \kappa=$ $0.403(p<0.001)$ for observers A (first time) and C, and $\kappa=0.377(p<0.001)$ for observers B and C.

\section{Discussion}

The success of a tendon healing firmly to its osseous attachment site is vital for continued musculoskeletal function after an injury. In this study, the relationship between changes on radiographs, fusion sign, and tendon-to-bone healing postoperatively for rotator cuff tears was investigated. The PPV of the fusion sign was high for all investigators, surgical procedures, and suture methods 
considered in this study. There were no significant differences between ORCR and ARCR or between the $\mathrm{SH}$ technique and the suture-bridging technique. Given these results, when the fusion sign is recognized, it is very likely that tendon-to-bone healing has occurred. Conversely, the interobserver reliability as indicated by the kappa value was not so high. The reason for why the kappa value was not significantly high is believed to be differences among the observers in the judgment standard of positivity. Although it was easy to judge an image that showed obvious osteolysis at the footprint as positive, there might be different criteria used by our observers to explain the degree of radiolucency. To increase the interobserver reliability, it is necessary to clarify the quantitative standard of evaluation and classification.

Tendon-to-bone healing is often confirmed by MRI or US [12]. However, MRI has the disadvantage of being expensive, available in only selected institutions, and time-consuming to perform. While US is less expensive than MRI, it also is not possible to administer in all facilities, and its use during outpatient care interferes with everyday medical treatment.

In contrast, $\mathrm{X}$-ray is superior to the aforementioned two imaging modalities in terms of being relatively inexpensive and easy to deploy in all clinics as a routine examination. Our findings are expected to provide a useful option for predicting tendon-to-bone healing with a simple X-ray finding, the so-called fusion sign.

Tendon-to-bone healing has not yet been clearly understood despite many studies. It usually occurs with the formation of fibrovascular interface tissue between the tendon and bone. Of note, there are two types of entheses at the tendon-to-bone junction: direct (fibrocartilage entheses) and indirect (fibrous entheses) [13] [14]. Therefore, it is necessary to investigate which type is demonstrated when the fusion sign occurs at the time of tendon-to-bone healing.

The present study has several limitations. First, there was no pathological investigation of the tissue at the tendon-to-bone healing site. With that said, what the fusion sign demonstrates will be clarified, it is difficult to perform in clinical patients with good postoperative cuff integrity. Second, we were unable to gather evidence of precisely when tendon-to-bone healing occurred. In the future, it will be necessary to examine when fusion sign occurs by investigating the $\mathrm{X}$-rays longitudinally; however, assessing whether there is a time discrepancy between the appearance of the fusion sign and actual tendon-to-bone healing will require future investigative work. Third, in the retear cases, verification of the retear site was not performed. The fusion sign may be positive in so-called type 2 retears (i.e., retear at the medial site) [15] because tendon-to-bone healing is obtained in this retear type. Fourth, this study was retrospective in nature. Therefore, the possibility of unintentional selection bias during the patient selection process could not be fully excluded.

\section{Conclusion}

This study investigated the relationship between changes on radiographs and 
tendon-to-bone healing after rotator cuff repair. Postoperatively, the presence of the fusion sign at the footprint of the humerus strongly suggests that tendon-to-bone healing has occurred.

\section{Conflicts of Interest}

The authors declare no conflicts of interest regarding the publication of this paper.

\section{References}

[1] Minagawa, H., Yamamoto, N., Abe, H., et al. (2013) Prevalence of Symptomatic and Asymptomatic Rotator Cuff Tears in the General Population: From Mass-Screening in One Village. Journal of Orthopaedics, 10, 8-12. https://doi.org/10.1016/j.jor.2013.01.008

[2] Tempelhof, S., Rupp, S. and Seil, R. (1999) Age-Related Prevalence of Rotator Cuff Tears in Asymptomatic Shoulders. Journal of Shoulder and Elbow Surgery, 8, 296-299. https://doi.org/10.1016/S1058-2746(99)90148-9

[3] DeOrio, J.K. (1984) Results of a Second Attempt at Surgical Repair of a Failed Initial Rotator-Cuff Repair. The Journal of Bone and Joint Surgery, 66, 563-567. https://doi.org/10.2106/00004623-198466040-00011

[4] Park, M.C., Cadet, E.R., Levine, W.N., et al. (2005) Tendon-to-Bone Pressure Distributions at a Repaired Rotator Cuff Footprint Using Transosseous Suture and Suture Anchor Fixation Techniques. American Journal of Sports Medicine, 33, 1154-1159. https://doi.org/10.1177/0363546504273053

[5] Funakoshi, T., Suenaga, N., Sano, H., et al. (2008) In Vitro and Finite Element Analysis of a Novel Rotator Cuff Fixation Technique. Journal of Shoulder and Elbow Surgery, 17, 986-992. https://doi.org/10.1016/j.jse.2008.06.002

[6] Taniguchi, N., Suenaga, N., Oizumi, N., et al. (2014) Surface-Holding Repair: An Original Arthroscopic Rotator Cuff Repair Technique. Journal of Shoulder and Elbow Surgery, 23, 620-627. https://doi.org/10.1016/j.jse.2014.01.007

[7] Ellman, H. (1987) Arthroscopic Subacromial Decompression: Analysis of One- to Three-Year Results. Arthroscopy, 3, 173-181.

https://doi.org/10.1016/S0749-8063(87)80061-0

[8] Ajrawat, P., Dwyer, T., Almasri, M., et al. (2019) Bone Marrow Stimulation Decreases Retear Rates after Primary Arthroscopic Rotator Cuff Repair: A Systematic Review and Meta-Analysis. Journal of Shoulder and Elbow Surgery, 28, 782-791. https://doi.org/10.1016/j.jse.2018.11.049

[9] Bilsel, K., Yildiz, F., Kapicioglu, M., et al. (2017) Efficacy of Bone Marrow-Stimulating Technique in Rotator Cuff Repair. Journal of Shoulder and Elbow Surgery, 26, 1360-1366. https://doi.org/10.1016/j.jse.2017.02.014

[10] Taniguchi, N., Suenaga, N., Oizumi, N., et al. (2015) Bone Marrow Stimulation at the Footprint of Arthroscopic Surface-Holding Repair Advances Cuff Repair Integrity. Journal of Shoulder and Elbow Surgery, 24, 860-866. https://doi.org/10.1016/j.jse.2014.09.031

[11] Sugaya, H., Maeda, K., Matsuki, K., et al. (2007) Repair Integrity and Functional Outcome after Arthroscopic Double-Row Rotator Cuff Repair. A Prospective Outcome Study. The Journal of Bone and Joint Surgery. American Volume, 89, 953-960. https://doi.org/10.2106/00004623-200705000-00006 
[12] Kluger, R., Bock, P., Mittlböck, M., et al. (2011) Long-Term Survivorship of Rotator Cuff Repairs Using Ultrasound and Magnetic Resonance Imaging Analysis. American Journal of Sports Medicine, 39, 2071-2081.

https://doi.org/10.1177/0363546511406395

[13] Gerber, C., Schneeberger, A.G., Perren, S.M., et al. (1999) Experimental Rotator Cuff Repair. A Preliminary Study. The Journal of Bone and Joint Surgery, 81, 1281-1290. https://doi.org/10.2106/00004623-199909000-00009

[14] Snyder, S.J. and Burns, J. (2009) Rotator Cuff Healing and the Bone Marrow "Crimson Duvet" from Clinical Observations to Science. Techniques in Shoulder \& Elbow Surgery, 10, 130-137. https://doi.org/10.1097/BTE.0b013e3181c2a940

[15] Cho, N.S., Yi, J.W., Lee, B.G., et al. (2010) Retear Patterns after Arthroscopic Rotator Cuff Repair: Single-Row versus Suture Bridge Technique. American Journal of Sports Medicine, 38, 664-671. https://doi.org/10.1177/0363546509350081 